Revue d'histoire de l'Amérique française

REVUE D.HISTOIRE DE L'AMÉRIQUE FRANÇAISE

\title{
L'origine acadienne de Moncton : Le Coude
}

\section{Raoul Dionne}

Volume 37, numéro 3, décembre 1983

URI : https://id.erudit.org/iderudit/304179ar

DOI : https://doi.org/10.7202/304179ar

Aller au sommaire du numéro

Éditeur(s)

Institut d'histoire de l'Amérique française

ISSN

0035-2357 (imprimé)

1492-1383 (numérique)

Découvrir la revue

Citer cet article

Dionne, R. (1983). L'origine acadienne de Moncton : Le Coude. Revue d'histoire de l'Amérique française, 37(3), 399-416. https://doi.org/10.7202/304179ar d'utilisation que vous pouvez consulter en ligne.

https://apropos.erudit.org/fr/usagers/politique-dutilisation/ 


\title{
L'ORIGINE ACADIENNE DE MONCTON LE COUDE
}

\author{
RAOUL DIONNE \\ Département d'histoire \\ Université de Moncton
}

Les origines françaises de la région de Moncton ont été longtemps oubliées. Pour la majorité des gens, l'histoire de Moncton commence avec l'arrivée des colons de la Pennsylvanie. Des familles, recrutées par une compagnie de colonisation, avaient fondé le township de Moncton vers 1766, à l'emplacement du ruisseau Hall. Après des débuts difficiles, cette colonie agricole s'adapta au commerce du temps, qui était axé sur l'exploitation des forêts, pour ensuite se donner une vocation particulière dans la construction des voiliers. L'élimination progressive des voiliers au profit des bateaux à vapeur provoqua une forte crise économique à Moncton. Mais la ville sut tirer parti de sa situation géographique à l'ère des chemins de fer.

Ces rapides observations nous amènent à conclure que le site de la ville présentait de nets avantages, puisque la population réussit à survivre en s'adaptant aux divers changements économiques. Mais, avant l'expérience du township de Moncton, il y a de fortes chances que les Indiens et les Français aient aussi utilisé cet endroit si avantageux comme lieu de peuplement.

Les populations s'enracinent généralement dans des milieux riches en ressources naturelles et bien reliés aux autres régions ; même s'il est possible d'y survivre isolé, on cherche en général à communiquer et à échanger avec d'autres collectivités. Et c'est précisément la facilité de communication qui rend intéressante la région de Moncton. Avant la création des chemins de fer, le réseau fluvial était une voie de communication extrêmement importante et Moncton était alors un carrefour pour les trajets vers le fleuve St-Jean, la baie de Shédiac ou la baie de Fundy.

Dans cette étude du Coude, nom que portait Moncton sous le Régime français, nous voulons examiner le milieu physique, les peuplements indien et acadien, et suivre l'évolution du Coude à travers l'histoire de l'Acadie. Mais avant d'aller plus loin dans l'analyse, voyons les sources et les études spécialisées sur l'histoire de la région.

Dans Une colonie féodale en Amérique : l'Acadie, Rameau de Saint-Père fournit beaucoup de renseignements sur le développement

RHAF, vol. 37, no 3, décembre 1983 
acadien de Chipoudy. Il a une grande admiration pour le meunier Pierre Thibodeau, pionnier des rives de la baie de Chipoudy. Rameau possède une excellente connaissance des familles acadiennes qui émigrèrent aux Trois-Rivières : Petitcodiac, Chipoudy et Memramcook. Cependant il passe sous silence le Coude, qui fait alors partie de la paroisse de Petitcodiac (Hillsborough) ${ }^{1}$.

Placide Gaudet au cours de ses nombreuses recherches historiques a vite constaté la nécessité de compléter Rameau. Dans une conférence prononcée lors du Congrès national des Acadiens, en 1927, il retraça l'histoire des Acadiens du Coude. C'est alors qu'il révéla l'histoire de la chapelle acadienne du Coude et qu'il raconta l'exploration archéologique de $1884^{2}$.

Plusieurs historiens anglais et acadiens ont poursuivi les recherches de Gaudet. Ganong ${ }^{3}$ et Webster ${ }^{4}$ ont publié les journaux des militaires anglais en poste au fort Cumberland, l'ancien Beauséjour. Cette documentation est d'une grande importance pour la période de la déportation des Acadiens.

Les Pères Pacifique de Valigny ${ }^{5}$ et Philias Bourgeois ${ }^{6}$ ont examiné la correspondance des missionnaires d'Acadie et ont trouvé quelques détails sur les églises, les chapelles, et les missionnaires qui desservirent les Trois-Rivières de la baie de Chipoudy.

En 1961, Adrien Arsenault publiait une synthèse des connaissances sur le Coude ${ }^{7}$. Cet article, qui parut dans les cahiers de la Société historique acadienne, relança les recherches sur l'histoire locale. Peu de temps après, Lloyd Machum publiait A History of Moncton (18551965 ) et Alex Pincombe remontait aux origines de la ville dans sa thèse de maitrise intitulée History of Monkton Township (1700-1875)9.

En 1968, avec la fondation du Centre d'études acadiennes de l'Université de Moncton, on entreprit le dépouillement de nouveaux documents. La publication d'un inventaire des sources documentaires de l'Acadie $^{10}$ remit à jour les documents de Desbarres, Deschamps et

\footnotetext{
1 F.E. Rameau de Saint-Père, Une colonie féodale en Amérique: l'Acadie (1604-1881) (2 vols., Paris, 1889).

2 Fonds Placide Gaudet, Centre d'études acadiennes, CEA, 1-27-7.

3 W.F. Ganong, «Report and Map of Major George Scott's expedition to remove the French from the Petitcodiac in 1758,» NBHS, Collections, 13 (1930): 97-114.

4 J.C. Webster, Journal de Boishébert (Shédiac, 1931). — The Forts of Chignecto (Shédiac, 1930). - Journals of Beauséjour (Sackville, 1937).

5 P. Pacifique de Valigny, Chroniques des plus anciennes églises de l'Acadie (Montréal, Écho de St-François, 1944).

6 Philias Bourgeois, Les Anciens missionnaires de l'Acadie (Shédiac, 1910).

7 Adrien Arsenault, «Les Acadiens aux Trois-Rivières,» SHA, 1 (1961): 18-27.

9 Alex Pincombe, «The History of Monkton Township (ca 1700-1875)», thèse de maitrise (University of New Brunswick, 1969).

10 Inventaire général des sources documentaires sur les Acadiens (Centre d'études acadiennes, Moncton, 1975).
} 
plusieurs autres. Régis Brun, recherchiste pour ce volume, contribua beaucoup à l'accroissement des connaissances sur les Acadiens d'après la déportation, période qu'on avait baptisée «d'enracinement dans le silence».

Vers 1979, Paul Surette entreprenait une étude approfondie sur Memramcook ${ }^{11}$, premier centre important du regroupement acadien. Cette étude clarifie les problèmes liés à la distribution des terres et explique l'acharnement que mit la famille Desbarres à garder les Acadiens dans un demi-servage seigneurial.

Dans une thèse récente ${ }^{12}$, Jean-Roch Cyr analyse systématiquement le retour des Acadiens dans le milieu urbain de Moncton. Il y découvre 9 Acadiens en 1851; 14 en 1861; 39 en 1871 et 363 en 1881 . Il examine les professions des habitants, leurs migrations et essaye de comprendre pourquoi les Acadiens ont boudé le développement industriel naissant. Il explique ce refus par le traumatisme du paysan acadien qui s'implantait en ville dans un milieu plutôt hostile.

La carte de la région de Moncton montre que la rivière Petitcodiac coule dans une direction SO-NE jusqu'à Moncton, pour ensuite faire un virage de presque $90^{\circ}$ avant de s'écouler vers la baie de Fundy. D'après l'orientation de la rivière, il serait plus logique qu'elle se déverse dans la baie de Shédiac. Les géographes expliquent que les fortes marées de la baie de Shepody ont probablement capté la rivière Petitcodiac et changé son cours. Cet angle dans la rivière Petitcodiac avait pris le nom de «Coude» avant 1760. Comme plusieurs noms français, il décrit bien la topographie du lieu.

Lorsqu'on venait de la baie de Fundy, il était facile de naviguer jusqu'au Coude et, par les portages ou par les rivières avoisinantes, de s'acheminer vers la baie de Shédiac ou le fleuve St-Jean. Le Coude pouvait donc jouer le rôle de carrefour ou de relais au cours des excursions fluviales.

Le capitaine Gauthier de l'Île St-Jean, par exemple, racontait que, pour se rendre à Québec, il avait fait en 1756 le trajet suivant:

De Chédaïque à la rivière Pécoudiak, c'est un portage de six lieues et beau chemin; il y a ladite rivière six à huit habitations françaises. Remonté ladite rivière/environ deux lieues, fait ensuite le portage nommé Ouaigesmock, aussi de six lieues, jusqu'à une autre rivière qui doit être celle de Chiamaristi: (Canaan)... L'on suivit cette rivière sur la glace jusqu'à Jemsek. ${ }^{.}{ }^{3}$

\footnotetext{
11 Paul Surette, Histoire des Trois-Rivières (1763-1806) (Société historique de la vallée de Memramcook, 1981).

${ }_{12}$ Jean-Roch Cyr, «Les Acadiens de Moncton: aspects d'histoire sociale, 1698-1881,» thèse de maîtrise (Université de Moncton, 1982).

${ }_{13}$ Rameau de St-Père, op. cit., II: 374.
} 
Les navigateurs français et anglais ont laissé également des observations sur le mascaret et sur la force des marées dans la rivière Petitcodiac. Gaspard Chaussegros de Lery décrit ainsi le phénomène:

Le courant monte avec tant de rapidité qu'au commencement de la marée, il se fait un volume d'eaux de deux à trois pieds d'épaisseur qu'un cheval au petit galop peut à peine suivre et le courant continue de mesmes au montant et baissant de la marée. ${ }^{14}$

La rivière Petitcodiac est navigable de son embouchure jusqu'à Moncton sur une distance de $35 \mathrm{~km}$, mais il faut s'ajuster à ses fortes marées.

Les premiers habitants de la région furent les Amérindiens Micmacs ${ }^{15}$. Ils occupaient un territoire d'environ $50000 \mathrm{mi}^{2}$, dans les trois provinces Maritimes, sauf le bassin de la rivière Saint-Jean, territoire des Malécites. Des Micmacs habitaient aussi le sud de la péninsule gaspésienne. Ils vivaient d'un peu d'agriculture, mais surtout de pêche, de cueillette et de chasse. Il est difficile d'évaluer leur nombre avant 1600 . Le Père Biard évaluait leur population à 3000 , mais il avait rencontré peu de groupements. La recherche de la nourriture était leur plus grande préoccupation, et les mois de février et de mars représentaient pour eux les plus difficiles de l'année. C'est surtout durant l'hiver qu'ils pénétraient à l'intérieur des terres afin d'y faire la chasse; alors que pendant cette saison, les Micmacs vivaient en petites communautés, ils se regroupaient en villages au bord de la mer pendant les mois d'avril à octobre. Ces villages de 200 à 300 habitants n'étaient pas permanents et se retrouvaient à divers endroits le long des côtes.

L'arrivée des Blancs dans le territoire micmac ruina rapidement l'équilibre et le style de vie des premiers habitants. Leur population fut décimée par les maladies européennes. Ils perdirent peu à peu leur indépendance quand ils acceptèrent les produits manufacturés; de même leur culture fut bouleversée par le christianisme. Pour obtenir des fusils, des marmites ou des couvertures de laine, ils durent changer leurs habitudes de chasse et s'ajuster à la traite des fourrures.

Peu de documents font allusion à l'existence d'Indiens dans la région du Coude. Mais on sait que le Père Biard est venu les évangéliser dans le bassin de Chignectou en 1612. La Vallière mentionne que son fils faisait la traite dans le bassin de Chipoudy, ce qui implique une présence indienne dans la région. Si l'on tient compte du fait que les orignaux et les castors se déplacent peu, et que les Micmacs pouvaient facilement les localiser, il est fort plausible que certains groupements

\footnotetext{
14 Chaussegros de Lery, Mémoire pour servir à la navigation des rivières Chipoudy, Pecoudiak, Memeramkouk, Tintamarre, Chekchacady et Beaubassin, APC, Coll. C 11, 196, fol. 207.

15 L.F.S. Upton, Micmacs and Colonists: Indian-White Relations in the Maritimes, 17131867 (Vancouver, 1979).
} 
indiens aient exploité le territoire de chasse des comtés d'Albert et de Westmorland. Partis des villages de Bouctouche, de Shédiac et de Beaubassin, quelques Micmacs devaient voyager et hiverner le long de la rivière Petitcodiac.

Le commerce entre les Blancs et les Micmacs remonte au début du XVIe siècle alors que les pêcheurs commencèrent à échanger des produits européens contre des fourrures. Ce commerce prit vite de l'importance, ce qui justifia la création de compagnies commerciales et de colonisation. Les Micmacs comprenaient mal pourquoi ces Européens voulaient des fourrures trop vieilles pour en faire des vêtements. C'est qu'ils ignoraient l'utilisation du poil dans la fabrication des feutres. Quoi qu'il en soit, ce commerce modifia les habitudes des Indiens. Dans le but d'obtenir plus de peaux, ils devinrent plus individualistes et fort dépendants des outils de chasse des Français. Ces derniers établirent plusieurs postes de traite à des endroits stratégiques, et la région du Coude aurait été un endroit idéal pour intercepter le passage des Indiens.

Les Français entretenaient des relations amicales avec les Micmacs. Plusieurs Français de la région de la Hève ont contracté des alliances matrimoniales avec des Indiennes, ce qui renforçait les liens d'amitié et garantissait la sécurité des Blancs. Plus tard les Indiens ont abrité plusieurs Acadiens lors de la déportation et ont soutenu les troupes de Boishébert au cours des expéditions militaires. Cependant, il y a peu de traces de métissage des deux races dans la région du Coude.

Sous le Régime français, les colons acadiens étaient principalement des agriculteurs. Contrairement aux habitants de la NouvelleFrance, ils ont peu utilisé le commerce des fourrures pour développer leur économie. Par ailleurs la pêche commerciale était entre les mains de métropolitains français, bretons, basques ou rochelais. Découvrant suffisamment de terres fertiles dans les marais, les Acadiens ont d'abord fondé leurs établissements agricoles autour de Port-Royal.

Les colons recrutés dans l'ouest de la France et au sud de la Loire étaient déjà initiés à la culture dans les marais. Ils savaient construire des digues pour récupérer des sols fertiles. Cette technique acadienne est fort bien décrite par le voyageur français Dièreville dans la relation de son voyage de 1699.

Il faut pour avoir des bleds déssécher les marais que la mer en pleine marée inonde de ses eaux, et qu'ils appellent les Terres Basses; celles-ci sont assez bonnes, mais quel travail pour les mettre en état d'être cultivées ! On arrête pas le cours de la mer aisément; cependant les Acadiens en viennent à bout par de puissantes digues qu'ils appellent des Aboiteaux et voici comment ils font; ils plantent cinq ou six rangs de gros arbres tous entiers aux endroits par où la mer entre dans les marais, et entre chaque rang ils cou- 
chent d'autres arbres de long les uns sur les autres et garnissent tous les vides si bien avec de la terre glaise bien battue, que l'eau n'y sçaurait plus passer. Ils ajustent au milieu de ces ouvrages un esseau de manière qu'il permet à la marée basse, à l'eau des marais de s'écouler par son impulsion, et défend à celle de la mer d'y entrer. ${ }^{16}$

Dièreville affirme ensuite que les belles moissons obtenues dès la seconde année valaient l'effort de ces constructions. Ce travail pénible et long exigeait une main-d'oeuvre nombreuse, ce qui amenait les colons à s'entraider et à entreprendre l'assèchement communautaire des marais.

Un tel système de culture avait aussi ses inconvénients, surtout celui de provoquer l'éparpillement de la population. Quand les ressources d'un marais devenaient insuffisantes pour soutenir une population grandissante, il fallait émigrer vers de nouvelles terres. Un patriarche partait alors avec ses fils, ses gendres, ses neveux et ses amis à la recherche d'un nouveau marais. C'est ainsi qu'après Port-Royal, de nouveaux centres furent fondés autour du bassin des Mines et de celui de Chignectou.

L'écrivain du roi, Gargas, faisait en 1687 une critique assez virulente des techniques acadiennes et proposait comme solution le défrichement des terres hautes. Il écrivait:

Il serait aussi bon de les obliger aux défrichements des terres hautes. La plupart des habitants comme au Port-Royal, aux Mines, ne s'amusent qu'à faire des levées dans les marais où ils sèment leur bled, ce qui leur porte beaucoup de préjudice pour plusieurs raisons, dont la première est que le bled qu'ils sèment dans leurs marais est d'un grain très petit qui rend à moitié son, et qui ne conserve pas; que les marées bien souvent crèvent leurs aboiteaux et inondent leurs terres, qui ne produisent plus de quelques années quand elles ont été abreuvées d'eau salée; que les réparations qu'ils sont obligés de faire annuellement leur coûtent beaucoup de dépenses et de peine; qu'ils courent le risque que lorsqu'ils sont prêts de faire une récolte, une marée ne leur enlève toutes leurs espérances, ce qui est dangereux, et que travaillant leur marais ils sont dans une nécessité de fourrage qui fait qu'ils ne peuvent pas nourrir beaucoup de bestiaux, tant pour le labeur que pour la nourriture des habitants. Au lieu que, s'ils défrichaient les terres hautes, ils n'auraient que la première peine, auraient de fort bon grain et assez de mauvais pour nourrir une quantité prodigieuse de bestiaux. ${ }^{17}$

Malgré les dangers soulignés par Gargas, les Acadiens ont continué à cultiver les terres basses et à élever bêtes à cornes et moutons. Ils

\footnotetext{
16 Sieur de Dièreville, Relation de voyage du Port-Royal de l'Acadie (1699), édité par J.C. Webster (Toronto, 1933), 258.

17 «État de la conduite que j'ay tenue pendant mon séjour au pays de l'Acadie,» manuscrit de Gargas de 1687.
} 
manquaient rarement de blé et réussissaient même à vendre les surplus aux marchands bostonnais. Il faut reconnaître cependant que le système des aboiteaux serait devenu tout à fait inadéquat si la population avait dépassé un certain seuil. Les Acadiens d'avant 1755 sont principalement connus comme des «défricheurs d'eau».

$\mathrm{Au}$ cours des migrations acadiennes, des établissements se sont enracinés dans la région de Moncton. Le sieur Michel Le Neuf de La Vallière, fils du gouverneur de Trois-Rivières, fonda un poste de traite dans l'isthme de Chignecto et obtint une seigneurie de dix lieues carrées à Beaubassin en 1676. Au cours de l'année 1687, il confia son domaine seigneurial à son gendre Claude Sébastien de Villieu et retourna en Nouvelle-France. Villieu et les fils de La Vallière firent la traite des fourrures autour de Beaubassin tout en poursuivant leur carrière militaire. Alexandre La Vallière, chargé de distribuer les présents aux Indiens en 1693, fut accusé par Louis XIV d'avoir employé à son profit la poudre destinée aux Micmacs. Ce geste était dangereux parce que l'alliance des Indiens se maintenait grâce à des présents, et avec le petit nombre de soldats postés en Acadie il fallait compter sur le renfort des guerriers indiens.

La seigneurie de Beaubassin fut également célèbre pour son procès de sorcellerie. Jean Campagna fut accusé de faire mourir par sortilège des hommes et des bestiaux de Beaubassin. Andrée Martin, la veuve de François Pellerin, déclara dans sa déposition que Jean Campagna «souffla dans l'oeil de son mari et qu'en peu de temps il aurait senti le mal qui lui montait à la tête et fut attaqué d'une grosse fièvre chaude» ${ }^{18}$. Campagna se vengea aussi sur les vaches de Roger Kessy et de son épouse Marie Poirier quand ils refusèrent de lui donner leur fille en mariage. Huit jours après les vaches étaient tombées malades, malgré les bénédictions du Père Claude. La Vallière, touché de compassion, menaça Jean Campagna «de lui passer son épée au travers du corps, s'il continuait à arriver accident aux bestiaux». Le lendemain matin tous les bestiaux étaient sur pied.

La seigneurie recevait ses colons de la Nouvelle-France et de PortRoyal; nous trouvons en 1686 les familles de Germain Girouard, de Pierre Morin, de Jean Mignault, de Jacques Cochu, de Michel Poirier, de Roger Quessy, de Germain Bourgeois, de Thomas Cormier et de quelques autres. En 1698 il y avait 174 habitants à Beaubassin. Les Acadiens n'aimaient pas beaucoup devenir des tenanciers de La Vallière qui leur vendait les marchandises à fort prix; il était inflexible sur les arrérages des rentes et faisait saisir leurs vaches quand ils ne pouvaient payer en blé. De plus, les Acadiens craignaient les incursions

18 Rameau, op. cit., II: 305. 
anglaises comme celle du Colonel Church, qui, en 1696, avait brûlé les maisons et massacré les bestiaux.

En 1685, l'intendant De Meulles de la Nouvelle-France avait constaté qu'autour de Beaubassin il y avait une grande quantité de prairies et vingt-deux habitations sur les petites éminences; mais il notait:

aucun de ces habitants à trois ou quatre corps de logis assez raisonnables pour la campagne, la plupart ont déjà de douze à quinze bêtes à cornes et même vingt, dix à douze cochons et autant de bêtes à laine. Ils ne se donnent pas la peine de les faire venir dans l'étable hors deux ou trois mois de l'année et lorsqu'ils en ont affaire pour les tuer, ce qui est cause qu'ils en perdent beaucoup par les chiens sauvages qui les mangent. Ils n'ont pas encore grande quantité de terres labourées.

La plupart des femmes font elles-mêmes des étamines dont elles s'habillent et leurs maris aussi, elles font presque toutes les bas pour leurs familles, et se passent d'en acheter; ils ne se servent tous que des souliers sauvages qu'ils font eux-mêmes. Il vient tous les ans dans ce lieu une barque anglaise, au mois d'avril, qui leur apporte le reste de leurs petites nécessités, qu'ils achètent pour des pelleteries qu'ils ont eues des sauvages: il s'y fait aussi de la toile de lin. Les bestiaux actuels sont d'une méchante espèce qu'il faudrait changer. ${ }^{19}$

Ce texte illustre bien le style de vie des Acadiens de la fin du XVII ${ }^{\mathrm{e}}$ siècle. De Meulles raconte également que les Acadiens donnaient aux Indiens des produits manufacturés en échange des fourrures. Le bateau d'avril dont parle De Meulles venait probablement de Boston car on sait que le marchand John Nelson, associé à son oncle Thomas Temple, a beaucoup négocié en Acadie. Il est étrange cependant de constater que ni De Meulles ni les recensements ne mentionnent les chevaux, et pourtant les Acadiens en faisaient usage ${ }^{20}$.

Dans le mouvement de colonisation de l'Acadie, Port-Royal resta jusqu'en 1710 la principale source de recrutement pour les nouveaux centres. Les anciennes familles acadiennes laissaient au fils aîné la ferme paternelle et le père conduisait ses jeunes fils vers les nouveaux établissements. Port-Royal et Beaubassin étaient aux deux extrémités de la Baie française, et la population pénétra par vagues successives jusqu'au fond des bassins environnants: - Bassin des Mines suivi de celui de Cobequid, - Bassin de Cumberland et celui de Shepody.

La baie de Chignecto pénètre du côté est dans le bassin Cumberland, lieu de la seigneurie de Beaubassin. Du côté nord, elle forme un genre d'entonnoir à deux branches au bout de la baie de Shepody. A

\footnotetext{
19 Rameau, op. cit., I: 172-174.

A.M. Clark, Acadia: The Geography of Early Nova Scotia to 1760 (Madison, 1968),
} 236. 
partir de 1698, des établissements acadiens se développèrent autour des rivières Chipoudy, Petitcodiac et Memramcook. Ces trois rivières avaient l'avantage de former des marais, terres de prédilection des Acadiens. Ces terres marécageuses ne possédaient pas l'étendue de ceux de Beaubassin, mais elles pouvaient accueillir des petites agglomérations d'Acadiens. On estime à environ 76000 acres les terres basses de la région.

La fondation de Chipoudy est décrite par Des Goutins dans une lettre du 15 novembre 1699:

Plusieurs habitants de Port-Royal, chargés de nombreuses familles, ayant découvert une rivière dans le fond de la baie française, appelée Chipoudy, en juillet 1698, auraient représenté à M. Villebon le dessein qu'ils avaient de s'y établir, ce qu'ils n'auraient osé faire sans sa permission; lequel le leur permit, et ils auraient exécuté et travaillé cet établissement tant l'automne dernier, ils ont même un moulin prêt à être transporté sur leurs terres; mais ils se sont vus arrêtés par une opposition de M. de Villieu au nom de La Vallière, son beau-père et de $M$. de Villebon, neveu du sieur de La Vallière, lequel se prétend seigneur de tout ce pays. ${ }^{21}$

Les limites des seigneuries étaient fort vagues, celles de La Vallière sont décrites dans un texte de 1702 comme étant situées entre le Cap Breton et l'île Percé, et comprenant la baie de Chignecto et le Cap Tourmentin. Louis XIV et ses ministres connaissaient mal la géographie du territoire et ne précisaient les limites qu'en cas de conflits.

Comme nous l'avons déjà mentionné, les Acadiens entreprenants ne voulaient pas devenir tenanciers ou métayers de La Vallière. C'est pourquoi ils cherchaient des marais à l'ouest de la Petitcodiac, croyant s'établir à l'extérieur de cette immense seigneurie. La monarchie française avait adopté le système seigneurial comme cadre du peuplement français et méthode de distribution des terres. Le seigneur habitant son manoir au milieu des fermiers remplissait les fonctions de chef social et de chef militaire auprès du petit peuple qui lui était attaché. Les rentes et les obligations des censitaires, précisées par contrat, étaient généralement peu lourdes en Acadie. Les premiers Français avaient connu des conditions beaucoup plus dures en Europe et acceptaient sans contestation les conditions seigneuriales. Mais après une génération ou deux, la mentalité changea rapidement. Les seigneurs, souvent absents, n'entretenaient pas de liens étroits avec leurs censitaires et pouvaient difficilement les défendre lors des raids anglais en Acadie. Ainsi, la population devenue acadienne s'habituait à compter sur ses propres ressources, et on l'accusait même d'avoir des tendances républicaines.

Pour Pierre Thibodeau de Port-Royal, il était normal de chercher de nouvelles terres en dehors du cadre seigneurial. Il n'avait pas besoin

\footnotetext{
21 Rameau, op. cit., II: 334.
} 
d'un officier français pour le diriger. De plus, il avait une nombreuse famille, dont sept fils à installer. Sa fille aînée avait épousé le sieur Desgoutins, l'administrateur civil de la colonie, ce qui lui donnait un protecteur influent. Avec quatre de ses fils et Pierre Gaudet, il explora la région des Trois-Rivières et fixa son choix sur Chipoudy qu'il fonda en juillet 1698. Guillaume Blanchard et ses deux fils explorèrent plus au nord et fondèrent Petitcodiac (Hillsborough) en 1699.

Les agents de La Vallière essayaient de bloquer les fondations indépendantes des Trois-Rivières, mais les Thibodeau, avec l'aide de Desgoutins, déposèrent une requête auprès du roi et supplièrent sa Majesté de leur «accorder à titre de fief deux lieues de front, au lieu où ils ont commencé leur établissement». Le ministre de la marine, Ponchartrain, ajouta au bas de cette lettre: «Défendre d'exécuter jusqu'à nouvel ordre, sauf les permis aux habitants de défricher. $\gg^{22}$

Sans toutefois que ledit sieur de La Vallière puisse déposséder les habitants de ladite province, qui se trouveront en possession des terres et héritages qu'ils occupent en ce territoire, et qu'ils cultivent, habitent et font valoir, ou font cultiver, conformément à l'arrêt du 20 mars 1703; à la charge seulement des censives et droits seigneuriaux envers ledit seigneur de La Vallière pour les terres qu'ils possèdent en ce territoire. ${ }^{23}$.

Cette malheureuse décision confirme l'intention royale de maintenir le système seigneurial en Acadie, ce qui explique le faible développement des Trois-Rivières avant 1715. De plus, l'arrêt de 1705 annexe toute la région des Trois-Rivières à la seigneurie de Beaubassin. Seul Mathieu Martin, le premier-né en Acadie, se vit accorder le rang de seigneur à Cobequid. Á l'aube du XVIII e siècle, les développements de colonisation se faisaient beaucoup mieux par le système des migrations dirigées par un patriarche d'expérience. À cette époque ni les seigneurs ni les compagnies commerciales n'avaient les moyens de recruter et d'établir de nouveaux colons français.

Il valait mieux créer de nouveaux espaces de développement et encourager les jeunes ménages acadiens à émigrer pour ne pas appauvrir le domaine paternel. Pierre Thibodeau et Guillaume Blanchard étaient des hommes entreprenants et assez à l'aise pour diriger et fonder des seigneuries familiales. Ils avaient créé des unités agraires viables et amené ensemble des hommes qui se respectaient et acceptaient le lourd travail communautaire nécessaire à l'édification et à l'entretien des aboiteaux. Le domaine de Mathieu Martin prospérait alors que les centres de Chipoudy et de Petitcodiac végétaient sous le contrôle de la seigneurie de La Vallière. Généralement, les solutions élaborées au

22 Rameau, II: 334.

23 Ibid., II: 337. 
pays étaient plus efficaces que celles qu'on fabriquait au loin dans le château de Versailles. Heureusement, le système seigneurial disparut sous l'administration de Louisbourg.

Le Coude, qui sera le prolongement des fondations de Chipoudy et de Petitcodiac, ne naîtra pas avant l'épuisement des terres cultivables plus au sud. La guerre de succession d'Espagne amena la chute et la capitulation de Port-Royal. Par le traité d'Utrecht de 1713, la France cédait à la Grande-Bretagne l'Acadie dans ses anciennes limites. Elle avait préféré conserver ses droits de pêche et ses îles productrices de sucre et de café dans les Antilles. La France ne s'intéressait guère à l'Acadie agricole. Mais elle réalisait la nécessité de protéger militairement l'entrée du golfe St-Laurent pour assurer la défense de la Nouvelle-France. On érigea la forteresse de Louisbourg et, par un jeu d'interprétations des traités on tenta de limiter les pertes à la péninsule de la Nouvelle-Écosse. De son côté, la Grande-Bretagne ignora sa nouvelle conquête, et se contenta de laisser une garnison à l'ancien PortRoyal, rebaptisé Annapolis Royal.

Malgré les efforts pour encourager les Acadiens à quitter la Nouvelle-Écosse, peu de familles émigrèrent au Cap-Breton, parce qu'elles n'aimaient pas les terres rocheuses à proximité de Louisbourg. Rameau dira de cette fondation: «...forteresse mal placée, mal conçue, mal construite, sans approvisionnements, sans avenir, impossible à secourir, impossible à défendre, qui coûta 30 millions et qui ne servit jamais à rien.» 24

Les Acadiens vécurent assez paisiblement en Nouvelle-Écosse jusque dans les années 1740, mais pendant la guerre de succession d'Autriche, les troupes françaises envahirent la Nouvelle-Écosse, et la neutralité des Acadiens fut mise à l'épreuve. Ceux qui collaborèrent avec les troupes françaises durent s'exiler, mais ce fut plutôt l'exception.

Cependant, la garnison anglaise avait été fortement menacée et le gouvernement britannique accepta les recommandations des gouverneurs d'établir un peuplement anglais et protestant capable de soutenir sans réserve les garnisons anglaises. On fonda Halifax en 1749 et on encouragea un meilleur contrôle du territoire en construisant des forts dans les villages acadiens. Le problème des limites territoriales n'ayant jamais été réglé, la France avait recréé une Acadie française au CapBreton, à l' 1 le St-Jean et au nord de la péninsule de la Nouvelle-Écosse. L'abbé Le Loutre, agent du gouvernement français et missionnaire des Micmacs, fonda dans la région de Beaubassin un centre d'accueil pour recevoir les émigrants acadiens, qui se sentaient beaucoup moins sûrs en Nouvelle-Écosse depuis la fondation de Halifax. Le gouvernement

\footnotetext{
24 Rameau, II: 134.
} 
anglais réclamait tout l'ancien territoire de l'Acadie et essaya de le reprendre en 1750. Les soldats du chevalier de la Corne résistèrent avec l'aide des Acadiens et des Indiens; ils établirent même une frontière française à la rivière Missagouèche en construisant les forts Beauséjour et Gaspereau. Les Acadiens installés au sud de la rivière Missagouèche se déplacèrent alors vers les régions de Beauséjour et des Trois-Rivières. Sous les ordres de Le Loutre, des Micmacs brûlèrent toutes les habitations françaises de Beaubassin laissées vacantes par cette migration. Le capitaine La Vallière raconte qu'en octobre 1750,
le fort anglais (Lawrence) s'est trouvé en état de recevoir des troupes. Le vingt-trois septembre, les familles des Planches, Wis- kok, Mencan, Nainpan, les Hébert, Menoudy, dont les habitants étaient déjà à la pointe à Beauséjour évacuèrent et passèrent sur notre terrain; les sauvages et Acadiens mirent le feu dans toutes les maisons et granges qui étoient pleines de bled et de fourage, ce qui a causé une grande dizette, et obligé à nourrir toutes ces familles aux dépens du Roy, ayant perdu même une grande partye de leurs bestiaux dans cette occasion et le reste pendant l'hyver n'ayant point eû de sel pour saller ce qu'ils avoient pu réchapper. ${ }^{25}$

Les Acadiens de la Nouvelle-Écosse se sont donc déplacés en grand nombre en 1750, mais le gouverneur Cornwallis refusait de laisser sortir les Acadiens des Mines et d'Annapolis, parce qu'ils étaient nécessaires au ravitaillement des troupes anglaises. Les villages des Trois-Rivières reçurent beaucoup d'émigrés et ces derniers furent installés le long de la rivière Petitcodiac. C'est probablement à cette époque que le Coude se développa et devint un établissement acadien, s'ajoutant ainsi à ceux de Chipoudy, Petitcodiac et Memramcook.

Le Coude, le centre de ce nouveau peuplement, comprenait des petits villages qui s'échelonnaient entre Salisbury et le Cran (aujourd'hui Stony Creek), en face de Dover. Les deux rives de la rivière Petitcodiac étaient habitées par des Acadiens exploitant les plaines alluviales du Moncton métropolitain. Placide Gaudet indique que le Coude portait aussi les noms de Terre Rouge et de La Chapelle. L'abbé Le Guerne avait fait construire une petite chapelle en bois rond près du site actuel du Parkview Motel. Placide Gaudet raconte «qu'un certain dimanche après-midi à l'automne de 1884 il visita les lieux avec des notables de la ville et découvrit une dizaine de pierres posées en forme de rectangle. C'était à n'en plus douter l'emplacement de la chapelle de la Terre Rouge» ${ }^{26}$.

Une lettre de l'abbé de l'Isle-Dieu, datée de 1754, indique «qu'il y a 200 familles sur les rivières de Chipoudy, Petitcodiac et Memram-

25 Journal de La Vallière, RAC, 1905, Série F, App. N, 325.

Placide Gaudet, Conférence de 1927, CEA, 1-27. 
cook qui n'ont pas de prêtres» ${ }^{27}$. Le Père Philias Bourgeois ajoute que l'abbé Le Guerne y était affecté depuis 1753, mais n'y résidait pas. Sur le plan démographique, on estime généralement que les familles acadiennes du temps formaient en moyenne des unités de six personnes. On arriverait à une population de 1200 personnes dans les Trois-Rivières vers 1755 . Il est cependant impossible d'obtenir des chiffres exacts pour la seule région du Coude. Il serait intéressant d'examiner les familles fondatrices du Coude et de ses environs. Rameau de Saint-Père explique assez bien la situation de 1714 à 1730. Il écrit que les familles acadiennes d'Annapolis, n'ayant plus confiance dans l'administration anglaise envoient de nombreux émigrants dans le fond de la baie française et à Chipoudy, croyant s'établir en dehors des limites anglaises. Chipoudy et Petitcodiac atteignent environ 170 personnes en 1730 . Vers 1740 , les deux frères Brossard, Joseph et Alexandre, mariés à deux filles de Michel Thibodeau, remontèrent la rivière Petitcodiac, Joseph jusqu'au Cran et Alexandre, au village des Blanchard.

Vers 1750 , plusieurs colons se retirèrent en amont du Coude et y fondèrent le village des Babineau, probablement le long de la rivière Coverdale $^{28}$. À cette époque des bateaux de patrouille anglais circulaient souvent du Cap Enragé au fort Lawrence, et Chipoudy était particulièrement vulnérable. De plus, les familles pionnières de ChipoudyPetitcodiac (Thibodeau, Blanchard, Martin, Pitre, Daigle, Savoie, Brossard et Babineau) avaient maintenant des enfants adultes à installer. Les familles étaient très nombreuses dans la région des TroisRivières. Les deux frères Brossard eurent à eux deux vingt-deux enfants. Rameau prétend que les centres de Beaubassin-Chipoudy enregistrèrent des augmentations de population de $11 \%$ par année.

Les habitants de la paroisse de Petitcodiac étaient établis des deux côtés de la rivière du même nom. Quand il y avait une petite concentration d'habitants, on parlait d'un village: village des Babineau, des Brossard-Beausoleil, des Amirault, des Pierres à Michel. Ces villages étaient très petits, comprenant environ deux à sept familles de même origine souvent liées par des mariages. La paroisse de Petitcodiac en 1752 comptait environ 352 personnes.

Dans le journal du major Scott, l'historien Ganong a retrouvé une ancienne carte de 1758 qui donne les noms déformés des habitants et des villages acadiens situés au nord de la rivière Petitcodiac. Les Beausoleil-Brossard habitant l'ouest de la ville à proximité de Salisbury, suivis vers l'est de Jean Boye, Sonier, Lalande dit Bonapetit à l'embouchure du lac Jones, La Chapelle près du Bore Park, Sylvain Breau

27 Rameau, II: 385.

28 Ganong, The Report and Map of Major George Scott, III. 
à Lewisville, Jacques Sonier à l'est du ruisseau Hall nommé alors ruisseau d'Acadie ${ }^{29}$.

Du côté de Riverview, en partant de l'ouest, Dodoff (probablement Simon Daroüet), Landry, Paul Thibodeau, Jean Aucoin, Toussaint Blanchard, près du Mud Creek, et Jean Babineau.

L'étude de l'origine de ces familles montre que les Breau, Landry et Sonier proviennent de la Rivière-aux-Canards de Grand-Pré, Jean Aucoin de Cobequid, et les Thibodeau de Port-Royal ou de Chipoudy. Ces colons peuvent revendiquer le titre de pionniers dans la région de Moncton.

La région de Petitcodiac échappa à la déportation de 1755 et contribua au mouvement de résistance du fort Beauséjour, qui capitula le 16 juin 1755; et à celui de Gaspereau qui tomba le lendemain. Charles Deschamps, sieur de Boishébert, commandant d'un petit poste à la rivière St-Jean, n'était pas en mesure de résister aux attaques anglaises. Menacé par le lieutenant-colonel Monkton, il brûla le fort de Saint-Jean et se retira au petit fort de Nerepis en amont du fleuve de Saint-Jean. En apprenant que les habitations de Beauséjour avaient été brûlées et que les habitants fuyaient vers le nord, il abandonna Nerepis au mois d'août et vint diriger les Acadiens de Petitcodiac.

À la fin d'août 1755, le major Frye brûla le village de Chipoudy et captura une trentaine de femmes et d'enfants. Le major Frye divisa ensuite ses troupes en deux groupes pour attaquer Petitcodiac et Memramcook. Boishébert, soutenu par les Acadiens et les Indiens, attaqua les troupes anglaises et tua le chirurgien March et vingt-cinq soldats. Malgré cette attaque surprise, les troupes de Frye réussirent à brûler une grande quantité de maisons et de granges. Le journal du colonel Monkton raconte cet événement.

Major Frye returns back with very bad success for having dividing his party. One of them was surprised by the enemy and lost 23 killed and taken. One officer killed and another wounded; however they burnt upwards to 300 houses and brought in about 30 women and children. ${ }^{30}$

Cette destruction laissait beaucoup d'Acadiens dans une grande misère. Et ce n'était là que le début de cette chasse à l'homme qui devait se poursuivre de 1755 à 1758.

Les Acadiens au nord de Petitcodiac ne connurent pas les destructions de 1755 et le Coude fut épargné. Après le départ de Monkton du fort Cumberland, le major Scott dirigea plusieurs expéditions dans les

\footnotetext{
29 Ibid., 106-114.

30 Webster, The Forts of Chignecto, 115.
} 
villages acadiens autour de Beauséjour et essaya en vain de capturer Boishébert dans les environs de Shédiac.

Pendant l'hiver 1755-56, Boishébert fut très actif pour protéger les survivants, et c'est également lui qui prit charge des 226 déportés d'Annapolis qui réussirent à ramener à St-Jean le bateau qui devait les conduire en Caroline. Il installa environ 1000 Acadiens à Miramichi pour les mettre à l'abri des attaques anglaises. L'intendant Bigot disait en 1755:

Monsieur de Boishébert et le Père Germain nous demandent des vivres, des effets, poudre, balles et plomb pour des familles qui sont refugiées dans les bois pour n'être pas prises... M. de Boishébert me marque qu'il a mille âmes dans les bois et qui y moureraient si je ne les secourais pas. ${ }^{31}$

Après un terrible hiver, au cours duquel 400 personnes moururent de faim et de froid, 200 survivants se rendirent à Québec. Dans une lettre de 1756, l'abbé Le Guerne raconte que les Pères Germain et de La Brosse, qui se tenaient dans des maisons au haut de Petitcodiac à quinze lieues de Beauséjour ont été épargnés d'une expédition de Monsieur Scott (en 1755) à cause de la quantité des glaces ou de la Providence ${ }^{32}$. Nous savons également que le Père La Brosse exerça son ministère «dans la maison du nommé Toussaint Blanchard», d'après le registre paroissial. La Brosse ajoute qu'à partir du 14 mars 1756 il utilisait une chapelle que les habitants de Chipoudy, refugiés dans les bois, avaient bâtie ${ }^{33}$. Cette petite chapelle desservait les deux cents fuyards groupés autour du village des Babineau au lieu que le Père La Brosse appelle l'île de Pecoudiak - la Terre Rouge ou le Coude.

L'année 1758 n'allait pas améliorer la situation en Acadie. La forteresse de Louisbourg tombe: les Acadiens de l'île St-Jean sont déportés alors que ceux du nord de la rivière Petitcodiac subissent de nouveaux raids anglais. Les Acadiens qui avaient évité la déportation de 1755 se trouvaient en grand nombre dans les Trois-Rivières: deux cent-cinquante familles, et de ce nombre soixante femmes dont les maris avaient été emmenés en Nouvelle-Angleterre selon l'abbé Le Guerne ${ }^{34}$.

L'abbé Le Guerne, qui desservait les Trois-Rivières et Tintamare, essaya de convaincre les Acadiens de quitter les lieux. Dans son plaidoyer, il disait:

La résolution où est l'anglais de ne plus souffrir d'Acadiens dans ces cantons, les menaces réitérées qu'il fait d'ammener tous ceux qu'il pourra atteindre... la grande difficulté où est le Canada...

\footnotetext{
31 RAC (1905): 349-50.

32 Ibid.: 347.

33 Fonds Placide Gaudet, CEA, 1-27-1.

34 RAC (1905): 349-50.
} 
et de l'extrême misère dont on est menacé font qu'il serait plus sage d'évacuer vers les français sans plus tarder. ${ }^{35}$

Dans le plan d'évacuation, Le Guerne veut conduire les Acadiens vers Shédiac et de là au Canada. Il propose des déplacements pendant l'hiver, parce que les Anglais sont bloqués par les glaces et qu'au printemps les portages sont impraticables. Il ne se sent plus capable de les tenir à l'abri des nouveaux raids anglais. Il dit que «les Acadiens ont toujours quelque prétexte pour sortir du bois et espèrent même semer au printemps. Sur ces entrefaits l'anglais vient, en prend quelques-uns et les emmène» ${ }^{36}$. Le Guerne ajoute qu'il n'y a plus de sécurité dans les TroisRivières, mais les Acadiens sont d'après lui «d'une irrésolution qui a de quoy surprendre un général». Le plus dur c'est qu'il faut dire adieu à son pays, à son habitation, à sa maison, abandonner les animaux et essuyer bien des misères avant de se rendre au Canada ${ }^{37}$.

Les Acadiens conservaient l'espoir qu'une flotte française reprendrait le territoire. En attendant, il fallait se bien cacher dans les bois. Malheureusement, les troupes anglaises capturaient assez souvent quelques Acadiens, ce qui provoquait beaucoup de tristesse et de peur. Mais lorsqu'on évitait les captures pendant un certain temps, il était facile de s'endormir dans une fausse tranquillité.

D'un autre côté, ceux qui acceptaient les déplacements vers la mer avaient aussi leurs problèmes. Il fallait hiverner sans réserves alimentaires et vivre à l'indienne dans des campements assez rudimentaires. Plusieurs Acadiens avaient du mal à survivre quand ils devaient se contenter uniquement de viande. Ils souffraient de troubles digestifs et de dysenterie, et plusieurs en mouraient.

Les Acadiens autour du Coude se croyaient en sécurité et continuaient à vivre presque normalement dans leur isolement. Mais vers la fin de juin 1758, le major Scott, commandant au fort Cumberland, envoya des détachements de troupes régulières et de Rangers pour capturer les Acadiens de Petitcodiac. La milice acadienne, sous le commandement de Beausoleil-Brossard et de Pierre Surette, leur opposa une vive résistance. Les bateaux anglais firent semblant de se retirer et profitèrent de la nuit pour débarquer les troupes près du Cran. Le lendemain, les bateaux remontèrent la rivière et lorsque les Acadiens s'avancèrent pour faire feu sur le bateau, les soldats cachés sortirent alors de la forêt et les Acadiens furent pris au piège. Scott relate:

Thirty of the enemy came down to meet the sloop and fired upon her. The Captain with the main body, who were within them on the shore under cover of the woods... surrounded them, took 
nine prisoners, killed and scalped three, drove fourteen into the river, ten of whom were drowned, four swam across the river and the rest made their escape. ${ }^{38}$

Selon Placide Gaudet, cette bataille a eu lieu au Cran, mais le rapport de Scott parle de la rive nord-est, ce qui désignerait plutôt la région de Pré-d'en-Haut.

Les Anglais connaissaient maintenant le dernier refuge des Acadiens au nord de la Petitcodiac et la dernière expédition eut lieu le 11 novembre 1758. De nuit, les bateaux anglais remontèrent la rivière jusqu'au Jonathan Creek. Profitant de la surprise, le lt Meech s'empara de quatre Acadiens et de douze femmes et enfants. Ces prisonniers fournirent les renseignements qui permirent aux troupes anglaises d'entreprendre la destruction systématique des installations acadiennes sur les deux rives de la Petitcodiac. Ganong dira des Rangers de Goreham qu'ils sont plus admirables pour leur efficacité que pour leur humanité. Les capitaines Goreham et Danks ont laissé une carte donnant les sites approximatifs des fermes acadiennes de la région.

Dans le journal de Scott, aux entrées du 15 et 16 novembre 1758 , il est écrit:

At 3 o'clock in the afternoon Capt. Goreham with 40 of the Rangers brought down the privateer schooner... about three leagues above the point - a Gard (Bore Park).

Captain Goreham reported that he had burnt all the houses from the head of the river down along the north side amounting to upwards of a hundred great and small with a very great quantity of corn and killed all the cattle and had taken one prisoner all the others having escaped in the woods. ${ }^{39}$

Sur la rive sud le capitaine Danks avait fait la même chose:

He had burnt all the buildings of every kind amounting to one hundred and twenty-four. He had also burnt a prodigeous quantity of grain which he found hid in several places in the back parts of the woods with all the hay \& killed all the cattle. ${ }^{40}$

C'était la fin du peuplement acadien dans la majeure partie des anciens centres. Plusieurs Acadiens tenteront de survivre dans les bois ou émigreront vers Gédaïque et Miramichi. Les survivants des TroisRivières, vaincus et épuisés, se rendront aux autorités du fort Cumberland en 1759. Emprisonnés dans les forts anglais ou gardés sous surveillance militaire, les Acadiens vivront dans des anciennes fermes; ils recevront de faibles rations et seront invités à prêter le serment d'allégeance pour s'intégrer à la colonie anglaise de la Nouvelle-Écosse.

\footnotetext{
38 Ganong, Major Scott's Expedition, 99.

39 Ibid., 103.

40 Ibid., 104.
} 
Plusieurs refuseront ce serment et quitteront le pays vers 1764 . Joseph Beausoleil-Brossard nolisa une goélette à Halifax et émigra avec plusieurs familles à Saint-Domingue. Mais ce groupe ne s'adapta pas aux conditions tropicales des Antilles et finit par s'installer en Louisiane. Les Acadiens autour des forts Cumberland et Edward finirent par accepter le statut de citoyens britanniques. Souvent leurs anciennes terres furent octroyées à de grands propriétaires anglais ou à des officiers de la Guerre de Sept ans. Sauf à Memramcook, où les Acadiens eurent la possibilité de reprendre la culture sur des terres de marais, ils obtinrent le plus souvent des terres sur la côte est, aux sols beaucoup moins fertiles. D'agriculteurs qu'ils étaient, les Acadiens deviendront assez souvent pêcheurs.

Les Acadiens vécurent dans l'isolement, en marge du développement de la province du Nouveau-Brunswick pendant près d'un siècle, avant de rétablir les institutions nécessaires à leur épanouissement et à leur survivance. 